\title{
RINGS FOR WHICH CERTAIN FLAT MODULES ARE PROJECTIVE
}

\author{
BY \\ S. H. COX, JR. AND R. L. PENDLETON
}

1. Introduction. All rings are assumed to have a unit element $1 \neq 0$, and all modules and ring homomorphisms are unitary.

Let $n$ be a nonnegative integer. A ring $R$ is said to be a left $A(n)$ ring if given any exact sequence $0 \rightarrow M \rightarrow E_{1} \rightarrow \cdots \rightarrow E_{n}$ of finitely generated left $R$-modules with $M$ flat and $E_{i}$ free for each $i$, then $M$ is projective. Commutative $A(0)$ rings have been studied by S. Endo [8], K. Mount [16] and D. Lazard [12]. In fact Lazard has obtained a characterization of commutative $A(0)$ rings in terms of the topology of the prime spectrum. Left $A(0)$ rings have also been studied by $\mathrm{F}$. Sandomierski and D. E. Smith [20]. S. Cox has proved that every commutative ring is an $A(2)$ ring [7, Theorem 2.9]. $A(1)$ rings have been considered by $\mathrm{M}$. Auslander, to whom the authors wish to express their gratitude for several helpful conversations.

The present paper is concerned primarily with commutative $A(1)$ rings.

In $\S 2$ we state those results which are valid for rings which may not be commutative. The arguments in this section are basically homological. Proposition 2.2, due to M. Auslander, states that if $R^{I}$ is a submodule of a flat right. $R$-module for any set $I$, then $R$ is a left $A(1)$ ring. A product of rings is a left $A(n)$ ring if and only if each factor is a left $A(n)$ ring; for $n=0$, the index set must be finite (Theorem 2.5).

Starting with $\$ 3$ we deal only with commutative rings; the arguments become more ideal-theoretic. A ring is an $A(1)$ ring if and only if each cyclic flat submodule of a free module is projective (Corollary 3.3); this statement is translated into ideal-theoretic language in Theorem 3.8. Self-injective rings, absolutely flat rings, and rings of Krull dimension zero are all $A(1)$ rings.

$\S 4$ is concerned with the stability of property $A(n)$ under various ring-theoretic constructions. $R$ is an $A(n)$ ring if and only if the ring of polynomials over $R$ in an arbitrary number of variables is an $A(n)$ ring (Theorem 4.3). If the associated reduced ring of $R$ is an $A(n)$ ring, then so is $R$ (Theorem 4.5); the converse is false for $n=1$ (Example 5.18), and hence there is no purely topological characterization of $A(1)$ rings. We define the connected component rings $\left\{R_{\sigma} \mid \sigma \in \Sigma\right\}$ of a ring $R$; these are flat $R$-modules, and if $\Sigma$ is finite, then $R=\prod R_{\sigma}$ and Theorem 2.5 applies. However

Received by the editors April 16, 1969 and, in revised form, October 2, 1969.

Copyright (C) 1970, American Mathematical Society 
if $\Sigma$ is infinite there is no good relation between property $A(n)$ for $R$ and property $A(n)$ for the connected component rings of $R$ (Examples 5.16 and 5.21).

$\S 5$ consists of eight counterexamples, four of which have been mentioned above. Example 5.1 is a noncommutative ring which is not a left $A(n)$ ring for any $n$. Three sufficient conditions for a ring $R$ to be an $A(1)$ ring are: (1) $R$ is an $A(0)$ ring; (2) $R$ is a $C$-ring, meaning that any exact sequence $0 \rightarrow M \rightarrow F \rightarrow N \rightarrow 0$ of finitely generated $R$-modules with $M$ flat and $F$ free splits; and (3) $R$ is a $D$-ring, meaning that for any index set $I, R^{I}$ is a submodule of a flat module. Examples $5.2,5.3$, and 5.6 show that these three conditions are logically independent and hence that none of them are necessary for $R$ to be an $A(1)$ ring.

Our notation is basically that of [3]; in addition we use "f.g." for finitely generated and "f.p." for finitely presented. $1_{S}$ is the identity function on a set $S$; $\approx$ stands for all kinds of isomorphism; $\delta_{i j}$ is the Kronecker delta; $Z$ is the ring of integers, and $\boldsymbol{N}$ is the set of positive integers.

2. General results. This section includes all those statements about $A(n)$ rings which we know to be valid for rings which are not necessarily commutative. Propositions 2.1 and 2.2, as well as the essential ideas in the proofs of 2.3 and 2.4 are all due to M. Auslander. Proposition 2.3 and Theorem 2.4 were first proved by $\mathrm{S}$. Endo [8] for the special case when $R$ is commutative and $T$ is a ring of quotients of $R$ with respect to a multiplicative system of regular elements.

2.1. Proposition. Let $R$ be a ring, $M$ a left $R$-module. For each set $I$, put $M^{I}=\prod_{i \in I} M$, and let $\sigma(M): R^{I} \otimes M \rightarrow M^{I}$ be the canonical homomorphism. Then

(i) $M$ is f.g. $\Leftrightarrow \sigma(M)$ is surjective, for all sets $I\left({ }^{1}\right)$.

(ii) $M$ is f.p. $\Leftrightarrow \sigma(M)$ is bijective, for all sets $I$.

Proof. First suppose $\sigma(M): R^{M} \otimes M \rightarrow M^{M}$ is surjective. Then $\exists f_{1}, \ldots$, $f_{k} \in R^{M}$, and $x_{1}, \ldots, x_{k} \in M$ such that $\sigma(M)\left(\sum f_{i} \otimes x_{i}\right)=1_{M}$. It follows that $x_{1}, \ldots, x_{k}$ generate $M$. Now let $E \rightarrow F \stackrel{u}{\rightarrow} M \rightarrow 0$ be exact with $E$ and $F$ free left modules. Note that $N^{I}$ is an exact functor of $N, \sigma$ is functorial, and $\sigma(H)$ is bijective whenever $H$ is f.g. and free. If $M$ is f.g., we take $F$ f.g., so $\sigma(F)$ bijective implies $\sigma(M)$ surjective. If $M$ is f.p., we take $E$ and $F$ f.g., so $\sigma(E), \sigma(F)$ bijective imply $\sigma(M)$ bijective. Finally, if $\sigma(M)$ is bijective for all sets $I$, we may take $F$ f.g., and let $K=\operatorname{ker}(u)$. Then $\sigma(M), \sigma(F)$ bijective imply $\sigma(K)$ surjective, so $K$ is f.g., so $M$ is f.p. Q.E.D.

2.2. Proposition. If for each set $I, R^{I}$ is a submodule of a flat right module, then $R$ is a left $A(1)$ ring.

Proof. Suppose $u: M \rightarrow E$ is an injective morphism of f.g. left modules, with $M$ flat and $E$ free. Let $I$ be a set and select an injective morphism $v: R^{I} \rightarrow N$,

(1) The related assertion in [3, $\$ 1$, Exercise 2a] is incorrect as worded. 
where $N$ is a flat right module. Since $1_{N} \otimes u$ and $v \otimes 1_{M}$ are injective and $\otimes$ is a bifunctor, $1_{\left(R^{I}\right)} \otimes u$ is injective. Thus, since $\sigma(E): R^{I} \otimes E \rightarrow E^{I}$ is bijective and $\sigma$ is functorial, $\sigma(M)$ is injective. Therefore $M$ is f.p. by Proposition 2.1 , so $M$ is projective [5, Chapter I, §2, Exercise 15]. Q.E.D.

The implication of Proposition 2.2 cannot be reversed (Example 5.6); moreover, the hypotheses of Proposition 2.2 do not imply that $R$ is a left $A(0)$ ring (Example 5.3).

2.3. Proposition. Let $c: R \rightarrow T$ be an injective ring homomorphism. If $M$ is a f.g. flat left $R$-module such that $T \otimes_{R} M$ is projective as a $T$-module, then $M$ is a projective $R$-module.

Proof. Let $I$ be a set, and consider the commutative diagram:

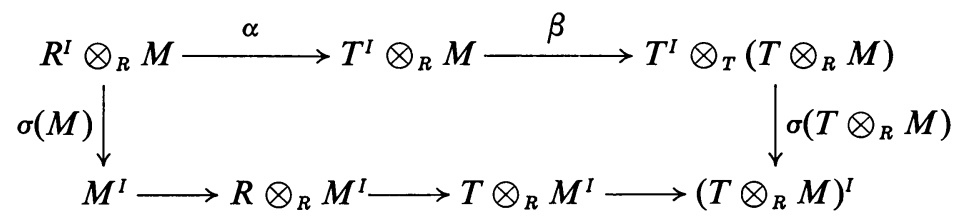

Since $M$ is $R$-flat, $\alpha=c^{I} \otimes 1_{M}$ is injective. $\beta$ is bijective, and since $T \otimes_{R} M$ is $T$ projective, $\sigma\left(T \otimes_{R} M\right)$ is bijective. Thus $\sigma(M)$ is injective, so by Proposition 2.1, $M$ is f.p. and hence projective. Q.E.D.

2.4. THEOREM. Let $c: R \rightarrow T$ be an injective ring homomorphism.

(i) If $T$ is a left $A(0)$ ring, so is $R$.

(ii) If $T$ is a left $A(n)$ ring ( $n \geqq 1)$, and $T$ is flat as a right $R$-module, then $R$ is a left $A(n)$ ring.

Proof. Let $0 \rightarrow M \rightarrow E_{1} \rightarrow \cdots \rightarrow E_{n}$ be an exact sequence of f.g. left $R$-modules, with $M$ flat and $E_{i}$ free, $\forall i$. Extend the scalar ring to $T$ to obtain a sequence of f.g. left $T$-modules $0 \rightarrow M \otimes_{R} T \rightarrow E_{1} \otimes_{R} T \rightarrow \cdots \rightarrow E_{n} \otimes_{R} T$ with $M \otimes_{R} T$ flat and $E_{i} \otimes_{R} T$ free, $\forall i$. For $n \geqq 1$ this sequence is exact by the flatness of $T$, while for $n=0$ the condition of exactness is vacuous. In either case, $M \otimes_{R} T$ is $T$-projective, so $M$ is $R$-projective by Proposition 2.3. Q.E.D.

2.5. THeOREM. Let $\left\{R_{i} \mid i \in I\right\}$ be a collection of rings, and $R=\prod R_{i}$. Then

(i) $R$ is a left $A(0)$ ring if and only if $I$ is finite and $R_{i}$ is a left $A(0)$ ring, $\forall i \in I$.

(ii) For $n \geqq 1, R$ is a left $A(n)$ ring if and only if $R_{i}$ is a left $A(n)$ ring, $\forall i \in I$.

Proof. Each $R_{i}$ is a projective left $R$-module, so it follows easily that if $R$ is an $A(n)$ ring, $n \geqq 0$, so is each $R_{i}$.

Now suppose each $R_{i}$ is a left $A(n)$ ring. For each $i \in I$, and each left $R$-module $N$, let $N_{i}=R_{i} \otimes_{R} N$. Let $0 \rightarrow M \rightarrow E_{1} \rightarrow \cdots \rightarrow E_{n}$ be an exact sequence of f.g. left $R$-modules with $M$ flat and $E_{j}$ free, $\forall j$. It is clear that $M_{i}$ is $R$-projective, $\forall i \in I$. If $I$ is finite, then $M=\oplus M_{i}$ is projective. Otherwise, let $n \geqq 1$, and consider the functor $T$ which associates to each left $R$-module $N$ the left $R$-module $\prod N_{i}$. 
It is easy to see that when restricted to the category of f.g. submodules of free modules, $T$ is naturally equivalent to the identity. Let $F \rightarrow M \rightarrow 0$ be exact with $F$ f.g. and free. By the remark above, this sequence may be identified with $\prod F_{i} \rightarrow \prod M_{i} \rightarrow 0$. But this latter sequence splits, since $F_{i} \rightarrow M_{i} \rightarrow 0$ splits for each $i \in I$. Thus $M$ is projective. Finally, if $R$ is a left $A(0)$ ring, define $e_{i}=\left(\delta_{i j}\right)_{j \in I}$, for each $i$, and let $A$ be the left ideal of $R$ generated by $\left\{e_{i} \mid i \in I\right\}$. Since $A$ is generated by idempotents, $R / A$ is flat by Corollary 3.5 and hence projective. Thus $A$ is f.g., which implies $I$ is finite. Q.E.D.

2.6. Proposition. If $n \geqq 0$ and $k \geqq 1$ are integers, then a ring $R$ is a left $A(n)$ ring if and only if $R_{k}$, the ring of $k \times k$ matrices over $R$, is a left $A(n)$ ring.

Proof. This is an immediate consequence of the results in [11].

For the case $n=0$, Proposition 2.6 is related to [20, Theorem 2.7].

3. Commutative $A(n)$ rings. From now on, all rings are assumed to be commutative. Thus our study of $A(n)$ rings reduces to the cases $n=0,1$. We reduce the problem to a consideration of cyclic modules, using the methods of Mount [16] applied to the invariant ideals of Auslander and Buchsbaum [1]. We then consider sufficient conditions for a ring to be an $A(1)$ ring.

Henceforth, in addition to the conventions listed in $§ 1$, we employ the terminology of commutative algebra as given in [5], except that we use $R_{S}$ instead of $S^{-1} R$ to denote the ring of quotients of a ring $R$ with respect to a multiplicative system $S$. We use $\operatorname{rad}[A]$ for the radical of an ideal $A$, and $\mathrm{Ann}_{R}(M)$ for the annihilator of the $R$-module $M$. By a prime component of an ideal $A$ in a ring $R$ we mean a minimal element of the set of prime ideals of $R$ containing $A$.

We wish to obtain a reduction from finitely generated modules to cyclic modules for the purpose of determining whether or not a ring is an $A(n)$ ring. For $n=0$, this reduction was first proved by Mount [16] using the Fitting invariants of a f.g. module, and later by Lazard, using his topological characterization of $A(0)$ rings. For our purposes it is more convenient to use the related invariants defined by Auslander and Buchsbaum [1].

If $R$ is a ring, $M$ a f.g. $R$-module, and $n \geqq 1$, we put $\alpha_{n}(M)=\operatorname{Ann}_{R}\left(\bigwedge^{n} M\right)$ and $F_{n}(M)=R / \alpha_{n}(M)\left({ }^{2}\right)$. The following result, similar to [16, Theorems 2 and 3], is an easy consequence of $[1]$.

3.1. Proposition. If $R$ is a ring and $M$ is a f.g. R-module, then $M$ is flat (resp. projective) if and only if $F_{n}(M)$ is flat (resp. projective) $\forall n \geqq 1$.

3.2. Lemma. Let $R$ be a ring, $M$ a f.g. flat $R$-module. If $M$ is a submodule of $a$ free module, then so is $F_{n}(M), \forall n \geqq 1$.

${ }^{(2)}$ If, for $j \geqq 0, f(j ; M)$ is the $j$ th Fitting invariant of $M$ as defined in [16], then it can be shown that $f(n-1 ; M)$ and $\prod_{i \geqq n} \alpha_{i}(M)$ have the same radical, although they are not equal in general. 
Proof. Let $v: F \rightarrow M$ be surjective and $u: M \rightarrow E$ be injective, where $E$ and $F$ are f.g. free $R$-modules. Put $w=u v$, and let $n \geqq 1$. Since $\bigwedge^{n} u$ is injective [14, Corollary to Theorem 2] and $\bigwedge^{n} v$ is surjective [4, $\S 5$, no. 7], we have $\alpha_{n}(M)$ $=\operatorname{Ann}\left(\bigwedge^{n} M\right)=\operatorname{Ann}\left(\bigwedge^{n} v\right)=\operatorname{Ann}\left(\bigwedge^{n} w\right)$. Thus

$$
0 \longrightarrow \alpha_{n}(M) \longrightarrow R \stackrel{f}{\longrightarrow} \operatorname{Hom}\left(\bigwedge^{n} F, \bigwedge^{n} E\right)
$$

is exact, where $f(1)=\bigwedge^{n} w$. Thus $F_{n}(M)=R / \alpha_{n}(M)$ is a submodule of a free module. Q.E.D.

3.3. Corollary. A ring $R$ is an $A(1)$ ring if and only if each cyclic flat submodule of a free module is projective.

At this point it is convenient to state various criteria for a cyclic module to be flat. If $R$ is a ring and $X=\operatorname{Spec}(R)$, let $D$ be the equivalence relation on $X$ generated by the relation of inclusion (see [12]). A subset $Y$ of $X$ is said to be $D$-closed if it is closed in the topology of $X$ and closed under the relation $D$. The following proposition can be found in [5] and [12].

3.4. Proposition. Let $R$ be a ring, $A$ an ideal of $R$, and put $S=1+A$, a multiplicative system in $R$. Let $i: A \rightarrow R$ and $\varphi: R \rightarrow R_{S}$ be the,canonical homomorphisms. Then the following are equivalent:

(1) $R / A$ is flat.

(2) $a \in A \Rightarrow \exists b \in A \cdot \ni \cdot a b=a$.

(3) $A=\operatorname{ker}(\varphi)$.

(4) If $P$ is a prime (resp., maximal) ideal of $R$ containing $A$, then $A_{P}=0$.

(5) $0 \rightarrow A \stackrel{\leftrightarrow}{\rightarrow} R \stackrel{\oplus}{\rightarrow} R_{S} \rightarrow 0$ is exact and $V(A)$ is $D$-closed.

Moreover, if $V(A)$ is $D$-closed and $B=\operatorname{ker}(\varphi)$, then $R / B$ is flat and $V(B)=V(A)$.

We note that the equivalence $(1) \Leftrightarrow(2)$ is valid for left ideals in noncommutative rings as well. Following [18], we say that $A$ is a *-ideal of $R$ if the conditions of 3.4 are satisfied.

3.5. Corollary. Let $R$ be a ring. If $A$ is an ideal of $R$ generated by a set $C$, and if $c \in C \Rightarrow \exists b \in A \cdot \ni \cdot c b=c$, then $A$ is $a^{*}$-ideal. In particular if $A$ is generated by $a$ set of idempotents, then $A$ is $a^{*}$-ideal.

Corollary 3.5 is valid for left ideals in noncommutative rings as well.

3.6. Corollary. Let $R$ be a ring. If $A$ and $B$ are *-ideals of $R$ such that $V(A)$ $=V(B)$, then $A=B$. Hence there is a 1-1 order reversing correspondence between *-ideals of $R$ and $D$-closed subsets of $\operatorname{Spec}(R)$.

Proof. Let $P \in \operatorname{Spec}(R)$. If $P \supseteq A$, then $A_{P}=B_{P}=0$ by 3.4(4); otherwise $A_{P}=B_{P}$ $=R_{P}$. Thus $A$ and $B$ induce the same sheaf of ideals over $\operatorname{Spec}(R)$, so $A=B$ $[9$, I.3.10]. (This may also be proved directly using 3.4(2).) The second assertion follows from the first, using 3.4. Q.E.D. 
3.7. Corollary. Let $R$ be a ring and $N=\operatorname{rad}$ [0], the nilradical of $R$. If $A$ is $a$ *-ideal of $R$, then $\operatorname{rad}[A]=A+N$.

Proof. Let $i: A+N \rightarrow \operatorname{rad}[A]$ be the canonical injection, and take $P \in \operatorname{Spec}(R)$. If $P$ q $A$, then $(A+N)_{P}=R_{P}=(\operatorname{rad}[A])_{P}$, so $i_{P}$ is surjective. If $P \supseteq A$, then $A_{P}=0$ by 3.4, so $(A+N)_{P}=N_{P}=(\operatorname{rad}[0])_{P}=\operatorname{rad}\left[A_{P}\right]=(\operatorname{rad}[A])_{P}$, and again $i_{P}$ is surjective. Hence $i$ is surjective. Q.E.D.

If $A$ is an ideal of a ring $R$, then $R / A$ is projective if and only if $A$ is generated by an idempotent of $R$ if and only if $V(A)$ is open and closed in $\operatorname{Spec}(R)$ [5, Chapter II, $\$ 4$, Proposition 15]. Moreover, $R / A$ can be imbedded in a free module if and only if $A=\operatorname{Ann}(B)$, where $B$ is some finitely generated ideal of $R$. Using these facts together with Proposition 3.4, Corollary 3.3 may be stated in more concrete terms.

3.8. Theorem. A ring $R$ is an $A(1)$ ring if and only if each *-ideal of $R$ which is the annihilator of a finitely generated ideal of $R$ is generated by an idempotent.

\subsection{Proposition. A self-injective ring is a $C$-ring.}

Proof. Let $R$ be a self-injective ring. Then dualizing is an exact functor, from which it follows easily that any f.g. submodule of a free module is reflexive. Furthermore, if $F$ is a flat module and $G$ is an injective module, then $\operatorname{Hom}(F, G)$ is injective [5, Chapter I, $\S 2$, Exercise 13]. Now let $M$ be a f.g. flat submodule of a free module. Then $M^{*}$ is injective, so $M^{*}$ is a direct summand of a f.g. free module, and hence $M^{*}$ is also flat. Thus $M \approx M^{* *}$ is injective. Q.E.D.

A ring $R$ is absolutely flat if every $R$-module is flat [5, Chapter I, $\S 2$, Exercise 17].

3.10. Proposition. An absolutely flat ring is a C-ring.

Proof. Suppose $0 \rightarrow M \rightarrow F \rightarrow N \rightarrow 0$ is exact with $M$ and $F$ f.g. Then $N$ is flat and f.p., so $N$ is projective [5, Chapter I, §2, Exercise 15] and hence the sequence splits. Q.E.D.

Further sufficient conditions for a ring to be a $C$-ring may be found in [2] and [13].

4. Stability. Next we consider questions of the following type: if $R_{1}$ and $R_{2}$ are rings related by some standard ring-theoretic construction, how does property $A(n)$ for $R_{1}$ relate to property $A(n)$ for $R_{2}$ ? In $\S 2$ we have already answered several questions of this type (Theorems 2.4, 2.5 and 2.6). Theorem 4.3 states that $R$ is an $A(n)$ ring if and only if the ring of polynomials (in arbitrarily many variables) over $R$ is an $A(n)$ ring. Theorem 4.5 states that if the associated reduced ring of a ring $R$ is an $A(1)$ ring, then so is $R$, although the converse is false.

Finally we discuss the connected component ideals $\left\{A_{\sigma} \mid \sigma \in \Sigma\right\}$ of a ring $R$, and the associated connected component rings $\left\{R_{\sigma}=R / A_{\sigma} \mid \sigma \in \Sigma\right\}$. The $R_{\sigma}$ 's are flat $R$-modules, and $R$ is imbedded in $\prod R_{\sigma}$. If $\operatorname{Spec}(R)$ has only finitely many connected components (in particular if $R$ is an $A(0)$ ring), then $R \approx \prod R_{\sigma}$ and Theorem 
2.5 applies. These results fail in general for rings whose spectra have infinitely many connected components.

Several other stability questions have negative answers.

Let $R$ be a ring. If $X$ is a set of indeterminates, we let $R[X]$ denote the ring of polynomials in the elements of $X$ with coefficients in $R$. If $f \in R[X]$, we let $A_{f}$ be the ideal of $R$ generated by the coefficients of $f$, and $\varphi(f)$ be the constant term of $f$, so that $\varphi: R[X] \rightarrow R$ is a ring homomorphism. The following proposition is the keystone of our arguments.

4.1. Proposition. Let $R$ be a ring, and let $f, g \in R[X]$. If $f g=0$, then

$$
\exists n>0 \cdot \ni \cdot A_{f}^{n} A_{g}=0 .
$$

Proof. Let $B=\operatorname{Ann}_{R}\left(A_{g}\right)$. We must prove $A_{f} \subseteq \operatorname{rad}[B]$. In case $R$ is a local ring with maximal ideal $P=\operatorname{rad}[B]$, the conclusion is immediate by induction on [17, Lemma 6.13, p. 17]. In general, let $P$ be a prime component of $B$ and localize at $P$ to reduce to the case above. Q.E.D.

Proposition 4.1 can also be proved by direct calculation.

4.2. Lemma. Let $R$ be a ring, $A a^{*}$-ideal of $R[X]$. Then:

(1) $A_{f} \subseteq A, \forall f \in A$.

(2) $A \cap R=\varphi(A)$.

(3) $A=\varphi(A) \cdot R[X]$.

Proof. (1) Let $f \in A$, and choose $g \in A$ so that $f(1-g)=0$. By Proposition 4.1, $A_{f} A_{(1-g)}^{n}=0$, for some $n>0$. Since the coefficients of $(1-g)^{n}$ belong to $A_{(1-g)}^{n}$, $A_{f}(1-g)^{n}=0$. Now $(1-g)^{n}=1-h$, for some $h \in A$, so $A_{f}=A_{f} h \subseteq A$.

(2) Clearly $A \cap R \subseteq \varphi(A)$. Let $f \in A$; then $\vec{\varphi}(f) \in A_{f} \subseteq A$ by (1), so $\varphi(A) \subseteq A \cap R$.

(3) Clearly $A \supseteq \varphi(A) \cdot R[X]$, by (2). Let $f \in A$; then $A_{f} \subseteq A \cap R=\varphi(A)$, so $f \in \varphi(A) \cdot R[X]$. Q.E.D.

Note in particular that Lemma 4.2 implies that the idempotents of $R[X]$ are precisely the idempotents of $R$.

4.3. TheOREM. Let $R$ be a ring. Then $R$ is an $A(n)$ ring if and only if $R[X]$ is an $A(n)$ ring.

Proof. The if part is a consequence of Theorem 2.4. Suppose $R$ is an $A(0)$ ring, and let $A$ be a *-ideal of $R[X]$. Then clearly $\varphi(A)$ is a *-ideal of $R$, so $\varphi(A)=R e$, for some idempotent $e \in R$. Thus by Lemma 4.2, $A=\varphi(A) \cdot R[X]=R[X] e$, and hence $R[X]$ is an $A(0)$ ring. Now suppose $R$ is an $A(1)$ ring. Let $F$ be a finite set of polynomials of $R[X]$, and put $A_{F}=\sum\left\{A_{f} \mid f \in F\right\}$, a f.g. ideal of $R$. If $A=\operatorname{Ann}_{R[X]}(F)$ is a ${ }^{*}$-ideal of $R[X]$, then by Lemma 4.2, $\varphi(A)=A \cap R=\operatorname{Ann}_{R}\left(A_{F}\right)$ is a *-ideal of $R$, so again $\varphi(A)=R e$ and $A=R[X] e$, where $e$ is an idempotent of $R$. Thus $R[X]$ is an $A(1)$ ring. Q.E.D.

In the case where $n=0$ and $X$ reduces to one element, Theorem 4.3 appears in [22, Corollary 2.2]. 
Given a ring $R$, we let $R_{\text {red }}$ denote the associated reduced ring, i.e., $R_{\text {red }}=R / \operatorname{rad}[0]$. Since $\operatorname{Spec}\left(R_{\mathrm{red}}\right) \approx \operatorname{Spec}(R)$ [9, Corollary 1.1.12], it follows from [12, Theorem 5.7] that $R$ is an $A(0)$ ring if and only if $R_{\text {red }}$ is an $A(0)$ ring.

4.4. Lemma. Let $B$ be an ideal of a ring $R$. If $A=\operatorname{Ann}(B)$ is $a{ }^{*}$-ideal of $R$, then $A=$ Ann $\left(B^{n}\right), \forall n>0$.

Proof. Let $n>0$, and suppose $x \in R$ with $x B^{n+1}=0$. Let $y \in B^{n}$. Then $x y B=0$ so $x y \in A$, so $\exists a \in A \cdot \ni \cdot x y=(x y) a=x(y a)=0$. Hence $x B^{n}=0$ and the lemma follows by induction. Q.E.D.

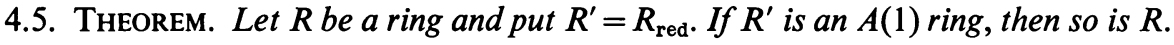

Proof. Let $\varphi: R \rightarrow R^{\prime}$ be the canonical homomorphism. Suppose $B$ is a f.g. ideal of $R$ such that $A=\operatorname{Ann}_{R}(B)$ is a *-ideal of $R$. Then $\varphi(B)$ is a f.g. ideal of $R^{\prime}$, and $\varphi(A)$ is a *-ideal of $R^{\prime}$. We claim $\varphi(A)=\operatorname{Ann}_{R^{\prime}}(\varphi(B))$. Let $x \in R$, with $\varphi(x) \varphi(B)$ $=0$. Then $x B \subseteq \operatorname{rad~[0],~and~since~} B$ is f.g., $x^{n} B^{n}=0$, for some $n>0$. Therefore $x^{n} \in \operatorname{Ann}\left(B^{n}\right)=A$ by Lemma 4.4 , so $x \in \operatorname{rad}[A]=A+\operatorname{rad}[0]$ by Corollary 3.7, and hence $\varphi(x) \in \varphi(A)$ as claimed. Under the homeomorphism Spec $(R) \approx \operatorname{Spec}\left(R^{\prime}\right)$, $V(A)$ corresponds to $V(\varphi(A))$. Since $R^{\prime}$ is an $A(1)$ ring, $V(\varphi(A))$ is open, so $V(A)$ is open in $\operatorname{Spec}(R)$. Thus $R / A$ is projective, so $R$ is an $A(1)$ ring. Q.E.D.

The converse to Theorem 4.5 is false (Example 5.18).

Let $R$ be a ring, and let $I=I(R)$ denote the set of idempotents of $R$. For $J \subseteq I$, let $A(J)$ denote the ideal of $R$ generated by $J$, and $V(J)=V(A(J)) \subseteq \operatorname{Spec}(R) . J$ is called proper if $A(J) \neq R$.

4.6. LEMMA. Let $R$ be a ring, $J \subseteq I(R)$.

(1) $J$ is a maximal proper set of idempotents of $R$ if and only if $J$ is proper and for any idempotent $e \in R$, either $e \in J$ or $1-e \in J$.

(2) If $J$ is a maximal proper set of idempotents of $R$, then:

(a) $A(J)=\{r e \mid r \in R, e \in J\}=\{x \in R \mid x=x e$, for some $e \in J\}$.

(b) $J=A(J) \cap I$.

(c) $V(J)$ is connected.

Proof. (1) The if part is clear. For the rest, it suffices to prove that if $e \in I$ and $e \notin J$, then $1-e \in A(J)$. Let $J^{\prime}=J \cup\{e\}$. By the maximality of $J, A\left(J^{\prime}\right)=R$, so $1=\sum r_{i} f_{i}+r e$, with $r_{i}, r \in R, f_{i} \in J$. Multiplication by $1-e$ yields the desired result.

(2a) follows easily from (1) and the fact that an ideal generated by a finite number of idempotents is generated by a single idempotent.

$(2 b)$ is clear from (1).

(2c) Suppose $x \in R$ and $x^{2}-x \in A(J)$. By (2a) $\exists e \in J \cdot \ni \cdot x^{2}-x=\left(x^{2}-x\right) e$, and hence $x(1-e)=x^{2}(1-e)=x^{2}(1-e)^{2}$ so $x(1-e) \in I$. If $x(1-e) \in J$, then $x=x(1-e)$ $+x e \in A(J)$, while if $1-x(1-e) \in J$, then $1-x=1-x(1-e)-x e \in A(J)$. Thus $R / A(J)$ has no nontrivial idempotents, so $V(J) \approx \operatorname{Spec}(R / A(J))$ is connected [5, Chapter II, $\S 4$, Corollary 2 to Proposition 15]. Q.E.D. 
4.7. Proposition. Let $R$ be a ring, $X=\operatorname{Spec}(R)$. If $A$ is an ideal of $R$ such that $Y=V(A)$ is a connected subset of $X$, and if $J(Y)=A \cap I(R)$, then $J(Y)$ is a maximal proper set of idempotents of $R$, and $V(J(Y))$ is the connected component of $X$ containing $Y$. The function $J \mapsto V(J)$ defines a bijective correspondence between maximal proper sets of idempotents of $R$ and connected components of $X$.

Proof. Suppose $Y=V(A)$ is connected. Clearly $J(Y)$ is proper. If $e \in I$, then either $e \in A$ or $1-e \in A$, since $R / A$ has no nontrivial idempotents, and hence $J(Y)$ is maximal. Now let $J$ be any maximal proper set of idempotents of $R$. By Lemma 4.6, $V(J)$ is connected, and since $V(J)=\bigcap\{V(e) \mid e \in J\}$ is an intersection of open and closed subsets of $X, V(J)$ is a connected component of $X$. The rest of the proposition follows easily from the statements above and Lemma $4.6(2 b)$. Q.E.D.

Much of Proposition 4.7 is purely topological; the sets $V(J)$, being nonempty intersections of maximal families of open and closed sets of $X$, are quasi-components of $X$. Lemma 4.6 (2c) establishes that the quasi-components of $X$ are connected, and hence coincide with the components of $X$ (cf. [10, pp. 46-47]).

If $R$ is a ring, $X=\operatorname{Spec}(R)$, and $\left\{Y_{\sigma} \mid \sigma \in \Sigma\right\}$ are the connected components of $X$, let $\left\{J_{\sigma} \mid \sigma \in \Sigma\right\}$ be the corresponding sets of idempotents, and put $A_{\sigma}=A\left(J_{\sigma}\right)$. The $A_{\sigma}$ 's are called the connected component ideals of $R$. By Corollaries 3.5 and 3.6, for each $\sigma \in \Sigma, A_{\sigma}$ is the unique *-ideal of $R \cdot \ni \cdot V\left(A_{\sigma}\right)=Y_{\sigma}$. Thus $A$ is a connected component ideal of $R$ if and only if $A$ is generated by idempotents and $V(A)$ is connected.

4.8. Proposition. If $\left\{A_{\sigma} \mid \sigma \in \Sigma\right\}$ are the connected component ideals of a ring $R$, then $\bigcap\left\{A_{\sigma} \mid \sigma \in \Sigma\right\}=0$, and hence the canonical homomorphism $R \rightarrow \prod R / A_{\sigma}$ is injective.

Proof. Let $x \in \bigcap A_{\sigma}$ and suppose Ann $(x)$ is proper. Select $P \in \operatorname{Spec}(R)$ so that Ann $(x) \subseteq P$, and choose $\sigma \in \Sigma$ so that $P \supseteq A_{\sigma}$. Since $A_{\sigma}$ is a *-ideal of $R$ and $x \in A_{\sigma}$, $\exists y \in A_{\sigma} \cdot \ni \cdot x(1+y)=0$. Thus $1+y \in$ Ann $(x) \subseteq P$, and $y \in A_{\sigma} \subseteq P$, a contradiction. Q.E.D.

If $\Sigma$ is finite, then $R \rightarrow \prod R / A_{\sigma}$ is bijective [5, Chapter II, $\S 4$, Proposition 15].

4.9. Corollary. Let $\left\{A_{\sigma} \mid \sigma \in \Sigma\right\}$ be the connected component ideals of a ring $R$. Then $R$ is an $A(0)$ ring if and only if $\Sigma$ is finite and $R / A_{\sigma}$ is an $A(0)$ ring, for all $\sigma \in \Sigma$. If $\Sigma$ is finite, then $R$ is an $A(1)$ ring if and only if $R / A_{\sigma}$ is an $A(1)$ ring, for all $\sigma \in \Sigma$.

Proof. If $R$ is an $A(0)$ ring, then $\left\{V\left(A_{\sigma}\right) \mid \sigma \in \Sigma\right\}$ forms an open partition of the compact space $\operatorname{Spec}(R)$ and hence $\Sigma$ is finite. The remainder of the corollary follows from the remark above and Theorem 2.5. Q.E.D.

When $\Sigma$ is infinite, the conclusions of Corollary 4.9 are not necessarily true. Indeed, Example 5.16 gives an $A(1)$ ring $R$ for which not all the rings $R / A_{\sigma}$ are 
$A(1)$ rings, and Example 5.21 gives a ring $R$ which is not an $A(1)$ ring and yet $R / A_{\sigma}$ is an $A(0)$ ring for each $\sigma \in \Sigma$.

We conclude this section with a brief discussion of some negative stability results. If $R$ is an $A(n)$ ring, and $A$ is an ideal of $R$, is $R / A$ an $A(n)$ ring? The answer is yes, trivially, if $A$ happens to be generated by an idempotent, by Theorem 2.5 . If $A$ is merely a *-ideal, the answer is in general no. Indeed, as we have remarked above, Example 5.16 gives an $A(1)$ ring $R$ and a connected component ideal $A$ such that $R / A$ is not an $A(1)$ ring. Attacking this question from a different point of view, suppose $R^{\prime}$ is a ring which is not an $A(1)$ ring (Example 5.21). Then we may write $R^{\prime}=R / A$, for some integral domain $R$ and some ideal $A \subseteq R$. Thus $R$ is an $A(0)$ ring, yet $R^{\prime}$ is not an $A(1)$ ring. Continuing, we may let $R^{\prime \prime}=R \times R^{\prime}$, which is not an $A(1)$ ring by Theorem 2.5. However the canonical morphism $R \rightarrow R \times R^{\prime}$ $=R^{\prime \prime}$ is injective and makes $R^{\prime \prime}$ into a finite $R$-algebra. This shows there is no hope for stability under integral ring extensions. Finally, we note that Example 5.17 gives an $A(0)$ ring $R$ and a multiplicative system $S \subseteq R$ such that $R_{S}$ is not an $A(1)$ ring.

5. Counterexamples. The conventions of $\$ 3$ remain in effect throughout this section, with the sole exception of Example 5.1. In addition, if $T$ is a subset of a ring $R$, then $(T)$ denotes the ideal of $R$ generated by $T$.

5.1. ExAmple. A ring $R$ such that $R$ is not a left $A(n)$ ring, $\forall n \geqq 0$.

Let $S$ denote the set of sequences $s=\left[s_{1}, \ldots, s_{n}\right]$ of positive integers such that $s_{i+1} \neq s_{i}+1, \forall i$. Let $k$ be a field, and define $R$ to be the $k$-vector space with basis $\{e\} \cup S \cup\left\{c_{\lambda} \mid \lambda \in N\right\} \cup\left\{d_{\lambda, s} \mid \lambda \in N, s \in S, s_{1} \neq 1\right\}$. Define multiplication on the basis elements of $R$ according to the following rules:

(1) $e x=x e=x, \forall x \in R$.

(2) Suppose $s=\left[s_{1}, \ldots, s_{n}\right]$ and $t=\left[t_{1}, \ldots, t_{m}\right] \in S$. Define $s t=0$ if $s_{n}+1=t_{1}$, and $s t=\left[s_{1}, \ldots, s_{n}, t_{1}, \ldots, t_{m}\right]$ otherwise.

(3) $s c_{\lambda}=s d_{\lambda, t}=0, \forall \lambda \in N, \forall s, t \in S$.

(4) $c_{\lambda} s=0$ if $s_{1}=1, c_{\lambda} s=d_{\lambda, s}$ otherwise.

(5) $c_{\lambda} c_{\mu}=\delta_{\lambda, \mu} c_{\mu}$.

(6) $c_{\lambda} d_{\mu, s}=\delta_{\lambda, \mu} d_{\mu, s}$.

(7) $d_{\lambda, s} t=0$ if $s_{n}+1=t_{1}$, and $d_{\lambda, s} t=d_{\lambda, s t}$ otherwise, where st is given by (2).

(8) $d_{\lambda, s} c_{\mu}=d_{\lambda, s} d_{\mu, t}=0, \forall \lambda, \mu \in N, \forall s, t \in S$.

This multiplication is associative on the basis elements of $R$, so $R$ becomes a $k$ algebra with identity $e$.

Put $a_{n}=[n] \in R, \forall n \in N$, and let $C$ be the left ideal of $R$ generated by $\left\{c_{\lambda} \mid \lambda \in N\right\}$. Then one verifies directly that $R a_{n}=$ left Ann $\left(a_{n+1}\right), n \geqq 1$, and that $C=$ left Ann $\left(a_{1}\right)$.

Put $M=R a_{1}$. Since $C$ is generated by idempotents, $C$ is a *-ideal of $R$ by Corollary 3.5 , and hence $M$ is a cyclic flat left $R$-module. Moreover, there is an exact sequence of left $R$-modules

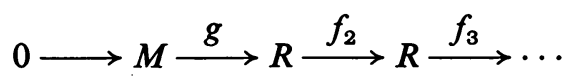


where $g$ is the canonical injection and $f_{n}(x)=x a_{n}, \forall n \geqq 2$. Finally, it is easy to see that $C$ is not f.g., and hence $M$ is not projective.

Thus $R$ is not a left $A(n)$ ring for any $n$. Q.E.D.

5.2. Example. A ring $R$ such that $R$ is a $D$-ring and an $A(0)$ ring but not a $C$-ring.

Let $R$ be any coherent domain which is not a field. Then $R$ is clearly an $A(0)$ ring and not a $C$-ring. $R$ is a $D$-ring by [6, Theorem 2.1].

5.3. Example. A ring $R$ such that $R$ is a $C$-ring and a $D$-ring but not an $A(0)$ ring.

Let $F$ be a finite field and $X$ an infinite extremely disconnected Boolean topological space (see [19]). Let $R$ be the ring of locally constant functions from $X$ into $F$. $R$ is absolutely flat [5, Chapter II, $\S 4$, Exercise 17b] and $R$ is an injective $R$-module [19, Corollary 24.2]. Thus $R$ is a $C$-ring by Proposition 3.9 or Proposition 3.10. For any index set $I, R^{I}$ is a projective $R$-module [19, Theorem 24.5], so $R$ is a $D$ ring. Now $\operatorname{Spec}(R) \approx X$ [5, Chapter II, $\S 4$, Exercise 17a], and since $X$ has infinitely many connected components, $R$ is not an $A(0)$ ring by Corollary 4.9. Q.E.D.

5.4. Proposition. Let $R$ be a local ring with maximal ideal $M$. Then the following are equivalent:

(1) $R$ is perfect.

(2) $M$ is $T$-nilpotent, i.e., for any sequence $\left\{f_{n}\right\}$ of elements of $M$, there exists a positive integer $m$ such that $f_{1} f_{2} \cdots f_{m}=0$.

(3) A direct limit of projective $R$-modules is projective.

(4) Every flat $R$-module is free.

Proof. (1), (2) and (3) are equivalent by [2, Theorem P]. (3) and (4) are equivalent by [14, Theorem 2] and [5, Chapter II, $\S 3$, Exercise 3c].

5.5. LEMMA. Let $R$ be a ring such that $R^{R}$ is a submodule of a free $R$-module. Then for any ideal $A \subseteq R$ there exists a f.g. ideal $B \subseteq R$ such that Ann $(A)=\operatorname{Ann}(B)$.

Proof. Let $R^{R} \subseteq F$, where $F$ is free with basis $S$. Define $x: R \rightarrow R$ by $x(r)=r$ if $r \in A, x(r)=0$ otherwise. Write $x=\sum a_{s} s \in F$, and let $B$ be the ideal generated by $\left\{a_{s} \mid s \in S\right\}$. Then Ann $(A)=\operatorname{Ann}(x)=\operatorname{Ann}(B)$, and $B$ is f.g.

5.6. ExAmple. A ring $R$ such that $R$ is a perfect local $A(0)$ ring and a $C$-ring but not a $D$-ring.

Let $R_{1}=k\left[X_{1}, X_{2}, \ldots\right]$ be the ring of polynomials in countably many indeterminates $X_{i}$ over a field $k$, and let $J=\left(\left\{X_{i} X_{j} \mid i \neq j\right\} \cup\left\{X_{i}^{i+1} \mid i \geqq 1\right\}\right)$. Then put $R=R_{1} / J$ and let $\varphi: R_{1} \rightarrow R$ be the canonical surjection. Put $x_{i}=\varphi\left(X_{i}\right)$. Clearly $R$ has but one prime ideal, namely $P=\left(x_{1}, x_{2}, \ldots\right)$. Thus $R$ is an $A(0)$ ring.

We claim that $P$ is $T$-nilpotent. Let $\left\{f_{n}\right\}$ be a sequence of elements of $P$. Using the defining relations we may select for each $n$ a representation $f_{n}=f_{n, 1}+\cdots$ $+f_{n, s(n)}$, where $f_{n, j}$ is a polynomial in $x_{j}$ alone, having constant term zero. It then 
follows that for $m=s(1)+1, f_{1} \cdots f_{m}=0$, which establishes the claim. Thus $R$ is a perfect local ring, by Proposition 5.4.

Let $B$ be a f.g. proper ideal of $R$. Then it is clear that for $n$ sufficiently large, $x_{n} B=0$. This proves first that $R$ is a $C$-ring by [2, Theorem 5.4 and Corollary 5.6] and second that $R$ is not a $D$-ring. For if $R$ were a $D$-ring, then by Lemma 5.5 there would exist a f.g. ideal $B$ such that Ann $(B)=$ Ann $(P)$, which would imply $x_{n} P=0$ for $n$ sufficiently large, an obvious contradiction. Q.E.D.

Our next example is an $A(1)$ ring $R$ such that for some connected component ideal $A, R / A$ is not an $A(1)$ ring. The example is easy to state; indeed

$$
R=Z\left[e_{1}, e_{2}, \ldots, y_{1}, y_{2}, \ldots\right]
$$

with defining relations $e_{i}^{2}=e_{i}, \forall i, 2 y_{i}=0, \forall i$, and $e_{i} y_{i}\left(1-y_{j}\right)=0, \forall i<j$. However the verification that $R$ satisfies the stated conditions is quite technical, and it is convenient to discuss some auxiliary theoretical results along the way.

Let $S$ be a set, $\Sigma=2^{S}$, and $\Sigma^{\prime}=\{\sigma \in \Sigma \mid \sigma$ is finite $\} . \Sigma^{\prime}$ is a semigroup under union. If $D$ is an integral domain, we put $D_{S}=D\left[\Sigma^{\prime}\right]$, the semigroup algebra.

5.7. Proposition. Let $S$ be $a$ set and $D$ a domain. Then:

(1) Given a commutative D-algebra $A$ and a function $f: S \rightarrow A$ such that $f(s)$ is idempotent, $\forall s \in S$, there is a unique D-algebra homomorphism $F: D_{S} \rightarrow A$ which extends $f$.

(2) Let $D[S]$ be the ring of polynomials in the elements of $S$, and

$$
I=\left(\left\{s^{2}-s \mid s \in S\right\}\right) .
$$

Then $D_{S}$ and $D[S] / I$ are canonically isomorphic D-algebras.

Proof. Both assertions are straightforward. Q.E.D.

Maintaining the preceding notation, suppose $\tau \in \Sigma$. The characteristic function $\chi_{\tau}$ induces a homomorphism $\theta_{\tau}: D_{S} \rightarrow D$, by Proposition 5.7. Specifically, if $\xi=\sum \xi_{\sigma} \sigma\left(\sigma \in \Sigma^{\prime}\right)$, then $\theta_{\tau}(\xi)=\sum \xi_{\sigma}(\sigma \subseteq \tau) . \theta_{\tau}$ is surjective, and $A_{\tau}=\operatorname{ker}\left(\theta_{\tau}\right)=$ $(\{s-1 \mid s \in \tau\} \cup\{s \mid s \notin \tau\})$. By the remark preceding Proposition 4.8, the $A_{\tau}$ 's are connected component ideals of $D_{S}$. On the other hand it is clear that the $A_{\tau}$ 's are precisely the minimal prime ideals of $D_{S}$. Thus the $A_{\tau}$ 's are all the connected component ideals of $D_{S}$. By Proposition 4.8 we have an injective ring homomorphism, $\theta: D_{S} \rightarrow D^{\Sigma}$, where $\theta(\xi)_{\tau}=\theta_{\tau}(\xi), \forall \tau \in \Sigma$.

5.8. Proposition. With notation as above,

$$
\operatorname{Im}(\theta)=\left\{d \in D^{\Sigma} \mid \exists \sigma \in \Sigma^{\prime} \cdot \ni \cdot d_{\tau}=d_{\tau \cap \sigma}, \forall \tau \in \Sigma\right\} .
$$

Proof. If $\xi=\sum \xi_{\rho} \rho\left(\rho \in \Sigma^{\prime}\right)$, we may take $\sigma=\bigcup\left\{\rho \mid \xi_{\rho} \neq 0\right\}$. Conversely, suppose $d \in D^{\Sigma}$ and $\sigma \in \Sigma^{\prime}$ with $d_{\tau}=d_{\tau \cap \sigma}, \forall \tau \in \Sigma$. Then define $\xi$ according to the rules $\xi_{\varnothing}=d_{\not}, \xi_{\rho}=0$ for $\rho \nsubseteq o$, and $\xi_{\rho}=d_{\rho}-\sum \xi_{\rho^{\prime}}\left(\rho^{\prime} \subseteq \rho, \rho^{\prime} \neq \rho\right)$ for $\rho \subseteq \sigma$. Then $\theta(\xi)=d$. Q.E.D. 
Next we study a family of rings $\left\{R^{\sigma} \mid \sigma \subseteq N\right\}$ which will turn out to be the connected component rings of the ring $R$ of our counterexample. The notation introduced hereafter will remain fixed until the proof of the counterexample is complete.

Let $R_{1}=Z[Y]=Z\left[Y_{1}, Y_{2}, \ldots\right]$ be the ring of polynomials in countably many variables with integer coefficients, and $R_{1}^{\prime}=R_{1} / 2 R_{1}=Z_{2}[Y]=Z_{2}\left[Y_{1}, Y_{2}, \ldots\right]$, and $\gamma: R_{1} \rightarrow R_{1}^{\prime}$ the canonical surjection. Put $M_{1}=\left(Y_{1}, Y_{2}, \ldots\right) \subseteq R_{1}$, and $M_{1}^{\prime}=\gamma\left(M_{1}\right)$. If $\sigma \subseteq N$, define $I^{\sigma}=\left(\left\{Y_{i}\left(1-Y_{j}\right) \mid i \in \sigma, j>i\right\} \cup\left\{2 Y_{i} \mid i \in N\right\}\right)$, and $J^{\sigma}=\gamma\left(I^{\sigma}\right)$. Let $\alpha_{\sigma}: R_{1} \rightarrow R_{1} / I^{\sigma}=R^{\sigma}$ be the canonical surjection, and put $\eta_{i}^{\sigma}=\alpha_{\sigma}\left(Y_{i}\right)$. When no confusion can result we shall drop the superscript $\sigma$, so that

$$
R^{\sigma}=Z[\eta]=Z\left[\eta_{1}, \eta_{2}, \ldots\right],
$$

with defining relations $\eta_{i}\left(1-\eta_{j}\right)=0, \forall i \in \sigma, \forall j>i$, and $2 \eta_{i}=0, \forall i \in N$.

5.9. LeMmA. $R^{\sigma} \approx Z+\left(M_{1}^{\prime} / J^{\sigma}\right)$, the ring obtained by adjoining $a$ unit to the Z-algebra $M_{1}^{\prime} / J^{\sigma}$.

Proof. Since $R_{1}=Z+M_{1}$, we have $R^{\sigma} \approx Z+\left(M_{1} / I^{\sigma}\right)$. But the homomorphism $\gamma: M_{1} \rightarrow M_{1}^{\prime}$ clearly induces an isomorphism $M_{1} / I^{\sigma} \approx M_{1}^{\prime} / J^{\sigma}$. Q.E.D.

Next we study the minimal prime ideals of $R^{\sigma}$. Let $P^{\sigma}=\left(\{2\} \cup\left\{Y_{i} \mid i \in \sigma\right\}\right)$, $p^{\sigma}=\alpha_{\sigma}\left(P^{\sigma}\right)$, and $Q^{\sigma}=\gamma\left(P^{\sigma}\right)$. If $i \in \sigma$, define

$$
P_{i}^{\sigma}=\left(\{2\} \cup\left\{Y_{j} \mid j \in \sigma, j<i\right\} \cup\left\{1-Y_{j} \mid j>i\right\}\right),
$$

$p_{i}^{\sigma}=\alpha_{\sigma}\left(P_{i}^{\sigma}\right)$, and $Q_{i}^{\sigma}=\gamma\left(P_{i}^{\sigma}\right)$. Also put $M^{\sigma}=\alpha_{\sigma}\left(M_{1}\right)$.

If $\sigma \neq \varnothing$ and $f \in Q^{\sigma}$, we define $i(f)=\min \left\{i \in \sigma \mid f \in\left(\left\{Y_{j} \mid j \in \sigma, j \leqq i\right\}\right)\right\}$. The proof of the following lemma is straightforward:

5.10. Lemma. If $\sigma \neq \varnothing$ and $f \in Q^{\sigma} \cap Q_{i(f)}^{\sigma}$, then $\exists g \in M_{1}^{\prime}$ such that $g \equiv f\left(\bmod J^{\sigma}\right)$ and $i(g)<i(f)$.

5.11. Proposition. (1) $J^{\sigma}$ is a radical ideal whose prime components are $Q^{\sigma}$ and $\left\{Q_{i}^{\sigma} \mid i \in \sigma\right\}$.

(2) If $f \in M_{1}^{\prime}$ and $f^{2}-f \in J^{\sigma}$, then $f \in J^{\sigma}$.

(3) $I^{\sigma}$ is a radical ideal whose prime components are $P^{\sigma},\left\{P_{i}^{\sigma} \mid i \in \sigma\right\}$, and $M_{1}$. (Except if $\sigma=N$, then $P^{\sigma} \supseteq M_{1}$.)

(4) $R^{\sigma}$ is a reduced ring whose minimal prime ideals are $p^{\sigma},\left\{p_{i}^{\sigma} \mid i \in \sigma\right\}$, and $M^{\sigma}$. (Except if $\sigma=N$, then $p^{\sigma} \supseteq M^{\sigma}$.)

(5) $\operatorname{Spec}\left(R^{\sigma}\right)$ is connected.

Proof. Throughout, the case $\sigma=\varnothing$ is clear.

(1) Clearly $Q^{\sigma}$ and $\left\{Q_{i}^{\sigma} \mid i \in \sigma\right\}$ are the prime components of $J^{\sigma}$. If $J^{\sigma}$ is not radical, then choose $f \in \operatorname{rad}\left[J^{\sigma}\right] \mid J^{\sigma}$ with $i(f)$ minimal and apply Lemma 5.10 to reach a contradiction.

(2) If $f \in M_{1}^{\prime}$ and $f^{2}-f \in J^{\sigma}$, then $f \in Q^{\sigma}$. If $f \notin J^{\sigma}$, then choose $f$ so that $i(f)$ is minimal. Again a contradiction results from Lemma 5.10. 
(3) and (4) follow from (1), and (5) is a consequence of (2), using Lemma 5.9. Q.E.D.

If $p$ is a prime ideal of $R^{\sigma}$, we say that $p$ is of Type I if $p \supseteq\left\{\eta_{i} \mid i \in \sigma\right\}$; otherwise $p$ is of Type II.

5.12. Proposition. (1) If $\sigma$ is infinite, then $\cap\left\{Q_{i}^{\sigma} \mid i \in \sigma\right\}=J^{\sigma}, \bigcap\left\{P_{i}^{\sigma} \mid i \in \sigma\right\}$ $=I^{\sigma}+2 R_{1}$, and $\bigcap\left\{p_{i}^{\sigma} \mid i \in \sigma\right\}=2 R^{\sigma}$.

(2) If $\sigma$ is finite, $\sigma \neq \varnothing$, and $k$ is the greatest element of $\sigma$, then $\bigcap\left\{Q_{i}^{\sigma} \mid i \in \sigma\right\}$ $=J^{\sigma}+\left(\left\{1-Y_{j} \mid j>k\right\}\right), \bigcap\left\{P_{i}^{\sigma} \mid i \in \sigma\right\}=I^{\sigma}+2 R_{1}+\left(\left\{1-Y_{j} \mid j>k\right\}\right)$, and $\bigcap\left\{p_{i}^{\sigma} \mid i \in \sigma\right\}$ $=2 R^{\sigma}+\left(\left\{1-\eta_{j} \mid j>k\right\}\right)$.

(3) Any two prime ideals of $R^{\sigma}$ of the same type are D-related.

(4) If $\sigma$ is finite, any two prime ideals of $R^{\sigma}$ are D-related; otherwise Type I prime ideals are not $D$-related to Type II prime ideals.

(5) $R^{\sigma}$ is an $A(1)$ ring if and only if $\sigma \neq N$.

Proof. (1) and (2) are straightforward calculations using Proposition 5.11.

(3) Clearly $p^{\sigma}$ and $M^{\sigma}$ are $D$-related, and if $i$ and $j$ are two adjacent elements of $\sigma$, with $i<j$, then $p_{i}^{\sigma}$ and $p_{j}^{\sigma}$ are both contained in the prime ideal of $R^{\sigma}$ generated by $\{2\} \cup\left\{\eta_{k} \mid k \leqq i\right\} \cup\left\{1-\eta_{k} \mid k>i\right\}$.

The proof of (4) is similar to that of (3).

(5) If $\sigma$ is finite, then $R^{\sigma}$ is in fact an $A(0)$ ring by (4). Now suppose $\sigma$ is infinite. Let $Y^{\sigma}$ denote the set of Type I prime ideals of $R^{\sigma}, Z^{\sigma}$ the set of Type II prime ideals of $R^{\sigma}$. It is easy to see that $Z^{\sigma}$ is not a closed subset of Spec $\left(R^{\sigma}\right)$; in fact $p^{\sigma}$ belongs to the closure of $Z^{\sigma}$. On the other hand, $Y^{\sigma}=V\left(p^{\sigma} \cap M^{\sigma}\right)$, and $p^{\sigma} \cap M^{\sigma}=$ $\left(\left\{\eta_{i} \mid i \in \sigma\right\}\right)$, which is a ${ }^{*}$-ideal by Corollary 3.5. If $\sigma=N$, then $p^{\sigma} \cap M^{\sigma}=M^{\sigma}=$ Ann (2), using Lemma 5.9, so $R^{\sigma}$ is not an $A(1)$ ring by Theorem 3.8. Finally, if $\sigma \neq N$, then it is straightforward to verify that $p^{\sigma} \cap M^{\sigma}$ is not the annihilator of a f.g. ideal, and hence by Theorem 3.8, $R^{\sigma}$ is an $A(1)$ ring. Q.E.D.

If $\rho \subseteq \sigma \subseteq N$, then $I^{\rho} \subseteq I^{\sigma}$, yielding a surjective homomorphism $\alpha_{\rho, \sigma}: R^{\rho} \rightarrow R^{\sigma}$.

5.13. Lemma. Suppose $\rho \subseteq \sigma \subseteq N, m \in N$, and $\rho \cap[1, m]=\sigma \cap[1, m]$, where $[1, m]=\{1,2, \ldots, m\}$. Then $J^{\sigma} \cap Z_{2}\left[Y_{1}, \ldots, Y_{m}\right] \subseteq J^{\rho}, I^{\sigma} \cap Z\left[Y_{1}, \ldots, Y_{m}\right] \subseteq I^{\rho}$, and $Z\left[\eta_{1}^{\rho}, \ldots, \eta_{m}^{\rho}\right] \cap \operatorname{ker}\left(\alpha_{\rho, \sigma}\right)=0$.

Proof. Straightforward calculation, using Proposition 5.12(1) and (2); the second and third assertions follow in order from the first. Q.E.D.

We are now ready to introduce the ring $R$. Begin with the polynomial ring $Z[E, Y]=Z\left[E_{1}, E_{2}, \ldots, Y_{1}, Y_{2}, \ldots\right]$ and its subring $Z[E]=Z\left[E_{1}, E_{2}, \ldots\right]$. Let $C$ be the ideal of $Z[E, Y]$ generated by $\left\{E_{i}^{2}-E_{i} \mid i \geqq 1\right\} \cup\left\{E_{i} Y_{i}\left(1-Y_{j}\right) \mid 1 \leqq i<j\right\}$ $\cup\left\{2 Y_{i} \mid i \geqq 1\right\}$, and $C_{0}=C \cap Z[E]$, which is the ideal of $Z[E]$ generated by $\left\{E_{i}^{2}-E_{i} \mid i \geqq 1\right\}$. Let $\lambda: Z[E, Y] \rightarrow Z[E, Y] / C=R$ be the canonical surjection, and put $e_{i}=\lambda\left(E_{i}\right), y_{i}=\lambda\left(Y_{i}\right)$, so $R=Z[e, y]=Z\left[e_{1}, e_{2}, \ldots, y_{1}, y_{2}, \ldots\right]$. Let $R_{0}=Z[e]$ $=Z\left[e_{1}, e_{2}, \ldots\right] \subseteq R$, so that $R_{0}=\lambda(Z[E]) \approx Z[E] / C_{0}$.

If $\sigma \subseteq N$, define $\psi_{\sigma}: Z[E, Y] \rightarrow Z[Y]=R_{1}$ by $\psi_{\sigma}\left(Y_{i}\right)=Y_{i}, \psi_{\sigma}\left(E_{i}\right)=1$ if $i \in \sigma$, and 
$\psi_{\sigma}\left(E_{i}\right)=0$ otherwise. Also let $\mu_{\sigma}: Z[E] \rightarrow Z$ denote the restriction of $\psi_{\sigma}$. Since $\psi_{\sigma}(C)=I^{\sigma}$ and $\mu_{\sigma}\left(C_{0}\right)=0$, there are induced surjective homomorphisms $\varphi_{\sigma}: R \rightarrow R^{\sigma}$ and $\theta_{\sigma}: R_{0} \rightarrow Z$, where $\varphi_{\sigma}\left(y_{i}\right)=\eta_{i}, \varphi_{\sigma}\left(e_{i}\right)=1$ if $i \in \sigma, \varphi_{\sigma}\left(e_{i}\right)=0$ if $i \notin \sigma$, and $\theta_{\sigma}$ is the restriction of $\varphi_{\sigma}$. Let $A^{\sigma}=\operatorname{ker}\left(\varphi_{\sigma}\right)=\left(\left\{1-e_{i} \mid i \in \sigma\right\} \cup\left\{e_{i} \mid i \notin \sigma\right\}\right)$, and $A_{0}^{\sigma}=\operatorname{ker}\left(\theta_{\sigma}\right)$ $=A^{\sigma} \cap R_{0}$.

Each $A^{\sigma}$ is a connected component ideal of $R$ by the remark preceding Proposition 4.8; since $\operatorname{Spec}(R)=\bigcup\left\{V\left(A^{\sigma}\right) \mid \sigma \subseteq N\right\},\left\{A^{\sigma} \mid \sigma \subseteq N\right\}$ is the set of all connected component ideals of $R$, and $R / A^{\sigma} \approx R^{\sigma}, \forall \sigma$. Similarly the $A_{0}^{\sigma}$ 's are the connected component ideals of $R_{0}$.

The proofs of the following two lemmas are straightforward.

5.14. Lemma. Given $F \in R, \sigma \subseteq N$, such that $\varphi_{\sigma}(F)=0$, there is a finite subset $\tau \subseteq \sigma$ such that $\varphi_{\rho}(F)=0$ whenever $\tau \subseteq \rho \subseteq \sigma$.

5.15. Lemma. Let $m \in N$. Suppose $F \in R$, where $F$ may be expressed as a polynomial in $e_{1}, \ldots, e_{m}, y_{1}, \ldots, y_{m}$. Let $\rho \subseteq \sigma \subseteq N$, with $\rho \cap[1, m]=\sigma \cap[1, m]$. Then $\varphi_{\sigma}(F)=\alpha_{\rho, \sigma}\left(\varphi_{\rho}(F)\right)$.

5.16. EXAmple. An $A(1)$ ring $R$ such that all but one of the connected component rings of $R$ are $A(1)$ rings.

$R$ is the ring defined after Lemma 5.13. By virtue of the preceding discussion, all that remains to be proved is that $R$ is an $A(1)$ ring. Suppose $F_{1}, \ldots, F_{n} \in R$ are such that $B=\operatorname{Ann}\left(F_{1}, \ldots, F_{n}\right)$ is a *-ideal of $R$. By Theorem 3.8 it suffices to find an idempotent $e \in R_{0}$ such that $V(B)=V(e)$.

Let $\sigma \subseteq N$. By Proposition 3.4, $R^{\sigma} \approx R_{S(\sigma)}$, where $S(\sigma)=1+A^{\sigma}$, and hence $B^{\sigma}$ $=\varphi_{\sigma}(B)=B_{S(\sigma)}=\operatorname{Ann}\left(\varphi_{\sigma}\left(F_{1}\right), \ldots, \varphi_{\sigma}\left(F_{n}\right)\right)$ is a ${ }^{*}$-ideal of $R^{\sigma}$. By Proposition 5.12, $B^{\sigma}=0$ or $B^{\sigma}=R^{\sigma}$ for $\sigma \neq N$, while for $\sigma=N, B^{\sigma}=0$ or $B^{\sigma}=R^{\sigma}$ or $B^{\sigma}=$ Ann (2). Define $n_{\sigma}=0$ if $B^{\sigma}=0, n_{\sigma}=1$ if $B^{\sigma}=R^{\sigma}, n_{\sigma}=2$ if $B^{\sigma}=$ Ann (2).

By Proposition 4.8, the homomorphisms $\left\{\varphi_{\sigma} \mid \sigma \subseteq N\right\}$ induce an injective homomorphism $\varphi: R \rightarrow \prod\left\{R^{\sigma} \mid \sigma \subseteq N\right\}$. Similarly $\theta: R_{0} \rightarrow Z^{\Sigma}$ is an injective homomorphism induced by $\left\{\theta_{\sigma} \mid \sigma \subseteq N\right\}$. (Here $\Sigma=2^{N}$.) $\theta$ is clearly the restriction of $\varphi$. The image of $\theta$ was described in Proposition 5.8. We claim that the tuple $\left(n_{\sigma}\right)_{\sigma \subseteq N}$ defined above belongs to the image of $\theta$.

Select an integer $m>0$ so that $F_{j} \in Z\left[e_{1}, \ldots, e_{m}, y_{1}, \ldots, y_{m}\right], \forall j$. Let $\sigma \subseteq N$, put $\rho=\sigma \cap[1, m]$, and claim $n_{\sigma}=n_{\rho}$. If $n_{\rho}=1$, then $\varphi_{\rho}\left(F_{j}\right)=0, \forall j$, so by Lemma 5.15, $\varphi_{\sigma}\left(F_{j}\right)=0, \forall j$, and hence $n_{\sigma}=1$. If $n_{\rho} \neq 1$, then $n_{\rho}=0$, since $\rho$ is finite. Suppose $n_{\sigma} \neq 0$. Then $\eta_{1} \in B^{\sigma}$, so $\varphi_{\sigma}\left(Y_{1} F_{j}\right)=0, \forall j$. By Lemma 5.14, there is a finite subset $\tau \subseteq \sigma$ so that $\varphi_{\mu}\left(Y_{1} F_{j}\right)=0, \forall j$, where $\mu=\tau \cup \rho$. Thus $\eta_{1} \in B^{\mu}$, so $B^{\mu}=R^{\mu}$, since $\mu \neq N$. Therefore $\varphi_{\mu}\left(F_{j}\right)=0, \forall j$. Let $f_{j}=\varphi_{\rho}\left(F_{j}\right)$. By Lemma $5.15, \alpha_{\rho, u}\left(f_{j}\right)=0, \forall j$, so by Lemma $5.13, f_{j}=0, \forall j$. But this implies $B^{\rho}=R^{\rho}$, so $n_{\rho}=1$, a contradiction, so $n_{\sigma}=0$. Thus by Proposition 5.8, there is $e \in R_{0} \cdot \ni \cdot \theta_{\sigma}(e)=n_{\sigma}, \forall \sigma \subseteq N$. Since $\theta$ is injective, $e$ is idempotent. Finally $V(B)=V(e)$ follows easily by intersecting each side with $V\left(A^{\sigma}\right), \forall \sigma . \quad$ Q.E.D. 
5.17. Example. A ring $R$ such that $R$ is an $A(0)$ ring, the Jacobson radical of $R$ does not contain a finite intersection of prime ideals, and there is a multiplicative system $S \subseteq R$ such that $R_{S}$ is not an $A(1)$ ring.

Let $R=Z\left[f, x_{1}, x_{2}, \ldots\right]$, with defining relations $f x_{i}\left(1-x_{j}\right)=0,1 \leqq i<j$, and $2 f x_{i}=0,1 \leqq i$. Put $S=\left\{f^{n} \mid n \geqq 1\right\}$. The proofs of the following assertions are either straightforward or similar to the arguments given in paragraphs 5.9 through 5.12. $R$ is a reduced ring; the minimal prime ideals of $R$ are $p=\left(x_{1}, x_{2}, \ldots\right), q=(f)$, and for each $i \geqq 1, p_{i}$, where $p_{i}=\left(\{2\} \cup\left\{x_{j} \mid j<i\right\} \cup\left\{1-x_{j} \mid j>i\right\}\right)$. Since the rings $R / p, R / q$, and $\left\{R / p_{i} \mid i \geqq 1\right\}$ all have Jacobson radical zero, $R$ has Jacobson radical zero. Thus the Jacobson radical of $R$ does not contain a finite intersection of prime ideals. The minimal prime ideals of $R$ are all $D$-related, so $R$ is an $A(0)$ ring with connected prime spectrum. Let $R^{\prime}$ be the ring $R^{N}$ of the preceding example, and let $T$ be an indeterminate over $R^{\prime}$. Then $R_{S} \approx R^{\prime}\left[T, T^{-1}\right.$, a flat $R^{\prime}$-module. By Theorem 2.4, $R_{S}$ is not an $A(1)$ ring. Q.E.D.

5.18. EXAMPLE. A coherent, self-injective $A(1)$ ring $R$ whose associated reduced ring is not an $A(1)$ ring.

Put $R_{0}=\prod_{x=1}^{\infty} Z$, let $p$ be a fixed positive prime, and define $R=\prod_{x=1}^{\infty} Z / p^{x}$. Let $\varphi: R_{0} \rightarrow R$ and $\alpha: R \rightarrow R^{\prime}=R_{\text {red }}$ be the natural surjections, and $\theta=\alpha \varphi$. For each $x, Z / p^{x}$ is self-injective [15], and hence $R$ is self-injective. For each $x, Z / p^{x}$ is a principal ideal ring. Thus any f.g. ideal of $R$, or the annihilator of any f.g. ideal of $R$, is principal, and hence $R$ is coherent. $R$ is an $A(1)$ ring by Proposition 3.9. It remains to show that $R^{\prime}$ is not an $A(1)$ ring. For this purpose it is convenient to regard $R^{\prime}=R_{0} / \operatorname{ker}(\theta)$. If $v$ denotes the $p$-adic valuation, then

$$
\operatorname{ker}(\theta)=\left\{f \in R_{0} \mid \exists n \cdot \ni \cdot n v(f(x)) \geqq x, \forall x\right\} .
$$

Define $g \in R_{0}$ by $g(x)=p, \forall x$. We claim Ann $(\theta(g))$ is a *-ideal of $R^{\prime}$ which is not generated by an idempotent.

Suppose $f \in R_{0}$, with $\theta(f) \in \operatorname{Ann}(\theta(g))$. Select an integer $n$ so that $n(v(f(x))+1)$ $=n v(f g(x)) \geqq x, \forall x$. Define $h \in R_{0}$ by $h(x)=f(x)$ for $x \geqq 2 n$, and $h(x)=1$ for $x<2 n$. Then $2 n(v(h(x) g(x))) \geqq x, \forall x$, so $\theta(h) \in$ Ann $(\theta(g))$, and $2 n(v(h(x) f(x)-f(x))) \geqq x$, $\forall x$, so $(\theta(h)-1) \theta(f)=0$. Thus Ann $(\theta(g))$ is a *-ideal of $R^{\prime}$.

Now suppose there is $e \in R_{0}$ such that $\operatorname{Ann}(\theta(g))=(\theta(e))$. Select $n$ so that $n(v(e(x))+1) \geqq x, \forall x$, and define $f \in R_{0}$ by $f(x)=1$ for $x \leqq n+1$ and $f(x)=p^{x}$ for $x>n+1$. Then $\theta(f) \in$ Ann $(\theta(g))$, so $\exists h \in R_{0}$ and $m>0$ so that $m(v(f(x)-h(x) e(x)))$ $\geqq x, \forall x$. Substituting $x=n+1$, we reach a contradiction. Q.E.D.

The following two propositions pave the way for our final example.

5.19. Proposition. Let $\left\{A_{\sigma} \mid \sigma \in \Sigma\right\}$ be the connected component ideals of a ring $R, N=\operatorname{rad}[0]$ be the nilradical of $R,\left\{N_{\sigma}=\operatorname{rad}\left[A_{\sigma}\right] / A_{\sigma} \mid \sigma \in \Sigma\right\}$ the nilradicals of the rings $R_{\sigma}=R / A_{\sigma}, R^{\prime}=R / N$, and $R_{\sigma}^{\prime}=R_{\sigma} / N_{\sigma}, \forall \sigma \in \Sigma$. Then $\left\{B_{\sigma}=\operatorname{rad}\left[A_{\sigma}\right] / N \mid \sigma \in \Sigma\right\}$ are the connected component ideals of $R^{\prime}$, and hence $\left(R^{\prime}\right)_{\sigma}=R_{\sigma}^{\prime}$.

Proof. Let $\varphi: R \rightarrow R^{\prime}$ be the canonical surjection. By Corollary 3.7, $\operatorname{rad}\left[A_{\sigma}\right]$ 
$=A_{\sigma}+N, \forall \sigma \in \Sigma$. Thus $B_{\sigma}=\varphi\left(\operatorname{rad}\left[A_{\sigma}\right]\right)=\varphi\left(A_{\sigma}\right)$ is a ${ }^{*}$-ideal of $R^{\prime}$, and $V\left(B_{\sigma}\right)$ corresponds to $V\left(A_{\sigma}\right)$ under the homeomorphism $\operatorname{Spec}(R) \approx \operatorname{Spec}\left(R^{\prime}\right)$. Q.E.D.

5.20. Proposition. Let $\left\{R_{i} \mid i \in I\right\}$ be a family of rings such that $\operatorname{Spec}\left(R_{i}\right)$ is connected, $\forall_{i} \in I$, and put $R=\prod R_{i}$. If $\mathscr{F}$ is any family of subsets of $I$, put $J(\mathscr{F})$ $=\left\{\chi_{S} \mid S \notin \mathscr{F}\right\}$, where $\chi_{S}$ is the characteristic function of the set $S$, and put $A(\mathscr{F})$ $=A(J(\mathscr{F}))$, the ideal of $R$ generated by $J(\mathscr{F})$. Then $J(\mathscr{F})$ is a maximal proper set of idempotents of $R$ if and only if $\mathscr{F}$ is an ultrafilter on $I$, and in that case, $A(\mathscr{F})$ $=\{f \in R \mid Z(f) \in \mathscr{F}\}$, where $Z(f)$ denotes the set of zeros of $f$.

Proof. Straightfoward.

5.21. Example. A ring $R^{\prime}$ which is not an $A(1)$ ring, and yet each connected component ring of $R^{\prime}$ is an $A(0)$ ring.

Let $R^{\prime}=R_{\text {red }}=R / N$, where $R$ is the ring of Example 5.18. By Proposition 5.20, the connected component ideals of $R$ are of the form $A(\mathscr{F})$, for some ultrafilter $\mathscr{F}$ on $N$, so by Proposition 5.19, the connected component ideals of $R^{\prime}$ are of the form $B(\mathscr{F})=\operatorname{rad}[A(\mathscr{F})] / N$. If $\mathscr{F}=\{S \subseteq N \mid x \in S\}$ is a fixed ultrafilter converging to $x \in N$, then it is easily verified that $A(\mathscr{F})=\{f \in R \mid f(x)=0\}$, and hence that $R^{\prime} / B(\mathscr{F}) \approx R / \operatorname{rad}[A(\mathscr{F})] \approx Z / p$, a field.

Now suppose $\mathscr{F}$ is a free ultrafilter on $N$, let $\alpha: R^{\prime} \rightarrow R^{\prime} \mid B(\mathscr{F})=R^{\prime \prime}$ be the canonical surjection and define $\psi=\alpha \theta: R_{0} \rightarrow R^{\prime \prime}$. We regard $\mathscr{F}$ as defining a finitely additive measure $\mu$ on $N$, where $\mu(S)=1$ if $S \in \mathscr{F}$ and $\mu(S)=0$ if $S \notin \mathscr{F}$. Using "a.e." for "almost everywhere," we have $\operatorname{ker}(\psi)=\left\{f \in R_{0} \mid(1 / x) v(f(x))\right.$ is bounded away from 0 , a.e.\}. It is then easy to show that $\operatorname{ker}(\psi)$ is a prime ideal of $R_{0}$, so that $R^{\prime \prime}$ is an integral domain. In point of fact it can actually be shown that for $f, g \in R_{0}$, $\psi(f) \in \psi(g) R^{\prime \prime}$ if and only if either $\psi(f)=0$ or $v(f(x)) \geqq v(g(x))$, a.e., from which it follows that $R^{\prime \prime}$ is a valuation ring.

In any case, if $\mathscr{F}$ is an ultrafilter on $N$, then the corresponding connected component ring $R^{\prime} / B(\mathscr{F})$ is an integral domain, and certainly an $A(0)$ ring. $R^{\prime}$ is not an $A(1)$ ring by Example 5.18. Q.E.D.

\section{REFERENCES}

1. M. Auslander and D. A. Buchsbaum, Invariant factors and two criteria for projectivity of modules, Trans. Amer. Math. Soc. 104 (1962), 516-522. MR 28 \#1215.

2. H. Bass, Finitistic dimension and a homological generalization of semi-primary rings, Trans. Amer. Math. Soc. 95 (1960), 466-488. MR 28 \#1212.

3. N. Bourbaki, Livre II: Algèbre. Chapter 2; Algèbre linéaire, 3rd ed., Actualités Sci. Indust., No. 1236, Hermann, Paris, 1962. MR 27 \#5765.

4. — Livre II: Algèbre. Chapter 3; Algèbre multilinéaire, Actualités Sci. Indust., No. 1044, Hermann, Paris, 1958. MR 30 \#3104.

5. - Algèbre commutative, Actualités Sci. Indust., Nos. 1290, 1293, 1308, Hermann, Paris, 1961-65. MR 30 \#2027; MR 36 \#146; MR 33 \#2660.

6. S. U. Chase, Direct products of modules, Trans. Amer. Math. Soc. 97 (1960), 457-473. MR 22 \#11017. 
7. S. H. Cox, Jr., Determinantal rank and flat modules, Proc. Amer. Math. Soc. 22 (1969), 104108. MR 39 \#2745.

8. S. Endo, On flat modules over commutative rings, J. Math. Soc. Japan 14 (1962), 284-291. MR 31 \#3475.

9. A. Grothendieck, Éléments de géométrie algébrique. I: Le langage des schémas, Inst. Hautes Études Sci. Publ. Math. No. 4 (1960). MR 29 \#1210.

10. J. Hocking and G. Young, Topology, Addison-Wesley, Reading, Mass., 1961. MR 23 \#A2857.

11. S. M. Kaye, Ring theoretic properties of matrix rings, Canad. Math. Bull. 10 (1967), 365-374. MR 35 \#4259.

12. D. Lazard, Disconnexités des spectres d'anneaux et des préschémas, Bull. Soc. Math. France 95 (1967), 95-108. MR 36 \#6422.

13. —, Algèbre symmétrique et platitude, C.R. Acad. Sci. Paris, Sér. A-B 264 (1967), A228-A230. MR 36 \#152.

14. — Sur les modules plats, C. R. Acad. Sci. Paris 258 (1964), 6313-6316. MR 29 \#5883.

15. L. S. Levy, Commutative rings whose homomorphic images are self-injective, Pacific J. Math. 18 (1966), 149-153. MR 33 \#2663.

16. K. Mount, Some remarks on Fitting's invariants, Pacific J. Math. 13 (1963), 1353-1357. MR 29 \#128.

17. M. Nagata, Local rings, Interscience Tracts in Pure and Appl. Math., no. 13, Interscience, New York, 1962. MR 27 \#5790.

18. B. L. Osofsky, Upper bounds on homological dimensions, Nagoya Math. J. 32 (1968), 315-322. MR 38 \#1128.

19. R. S. Pierce, Modules over commutative regular rings, Mem. Amer. Math. Soc. No. 70 (1967). MR 36 \#151.

20. F. L. Sandomierski and D. E. Smith, Rings for which finitely generated flat modules are projective, (to appear).

21. W. V. Vasconcelos, On finitely generated flat modules, Trans. Amer. Math. Soc. 138 (1969), 505-512. MR 39 \#199.

22. - On projective modules of finite rank, (to appear).

Louisiana State University, Baton Rouge, Louisiana 70803 